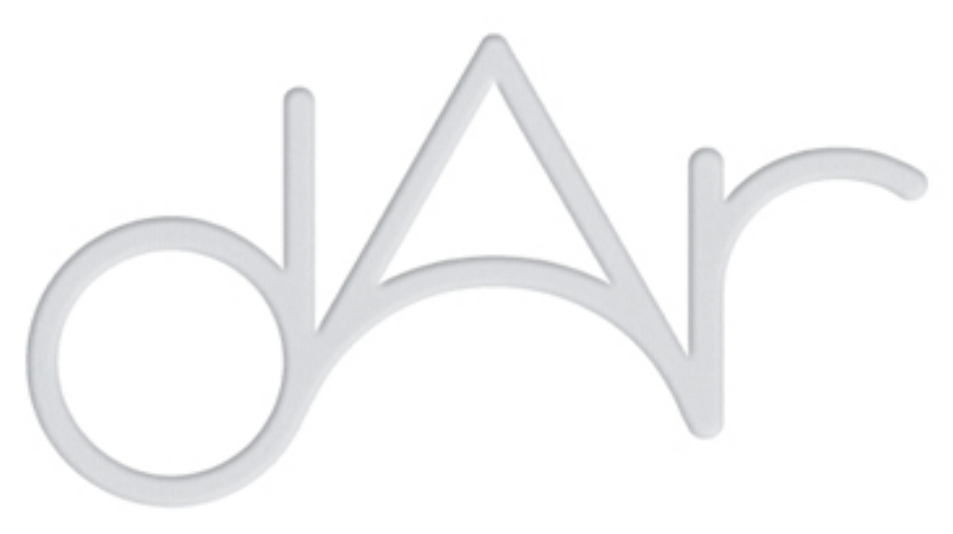

\title{
Le paysage oasien entre perception et réalité
}

Autor(es): Bouzahar, Soumia

Publicado por: Imprensa da Universidade de Coimbra

URL

persistente: URI:http://hdl.handle.net/10316.2/44426

DOI: $\quad$ DOI:https://doi.org/10.14195/2182-844X_5_5

Accessed : $\quad$ 26-Apr-2023 05:55:50

A navegação consulta e descarregamento dos títulos inseridos nas Bibliotecas Digitais UC Digitalis, UC Pombalina e UC Impactum, pressupõem a aceitação plena e sem reservas dos Termos e Condições de Uso destas Bibliotecas Digitais, disponíveis em https://digitalis.uc.pt/pt-pt/termos.

Conforme exposto nos referidos Termos e Condições de Uso, o descarregamento de títulos de acesso restrito requer uma licença válida de autorização devendo o utilizador aceder ao(s) documento(s) a partir de um endereço de IP da instituição detentora da supramencionada licença.

Ao utilizador é apenas permitido o descarregamento para uso pessoal, pelo que o emprego do(s) título(s) descarregado(s) para outro fim, designadamente comercial, carece de autorização do respetivo autor ou editor da obra.

Na medida em que todas as obras da UC Digitalis se encontram protegidas pelo Código do Direito de Autor e Direitos Conexos e demais legislação aplicável, toda a cópia, parcial ou total, deste documento, nos casos em que é legalmente admitida, deverá conter ou fazer-se acompanhar por este aviso. 

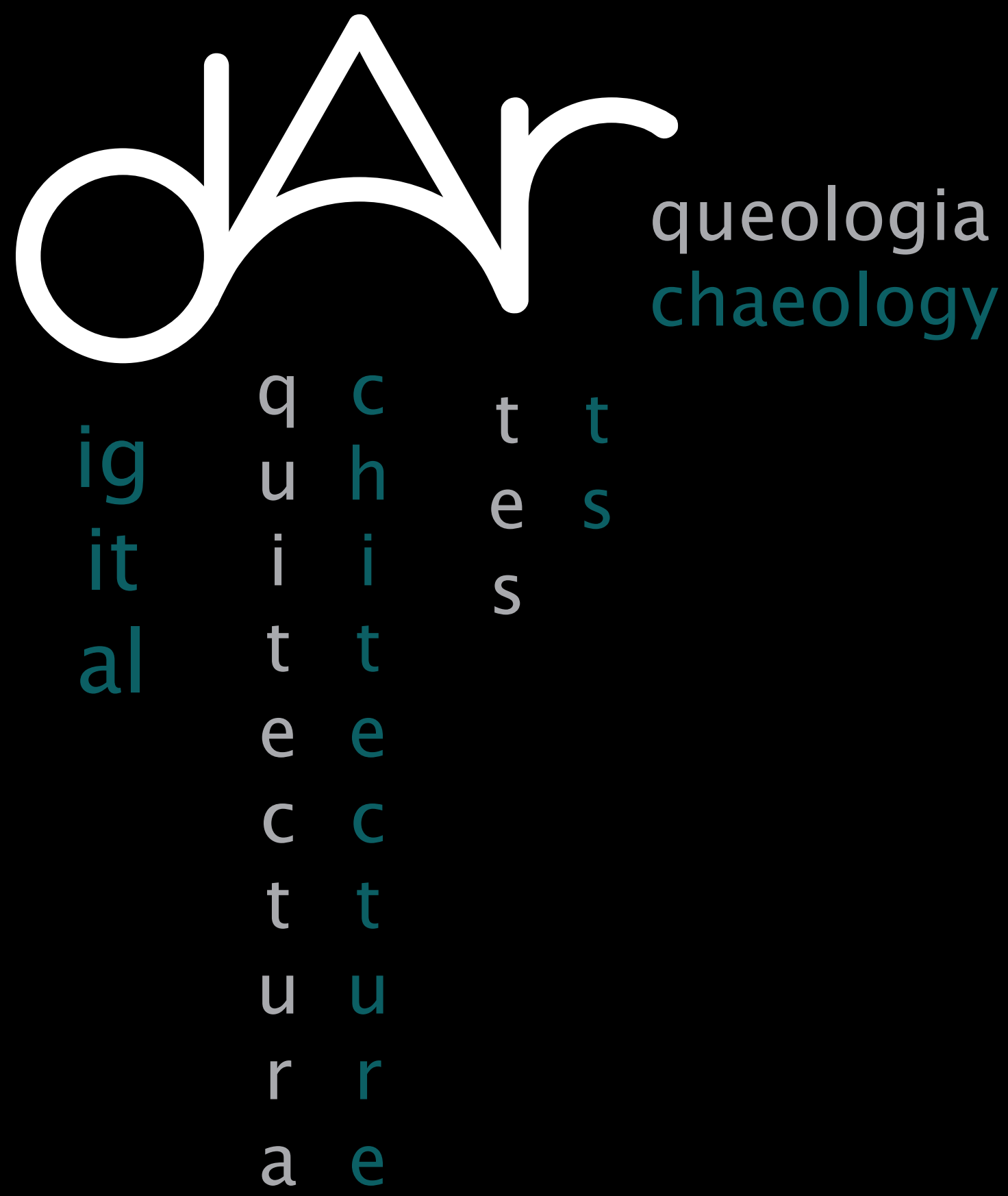

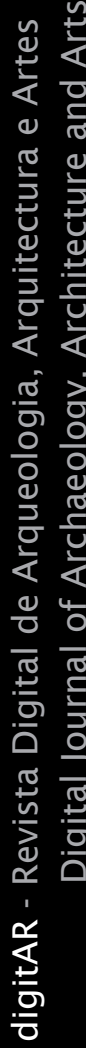




\title{
LE PAYSAGE OASIEN ENTRE PERCEPTION ET RÉALITÉ
}

\author{
Dr. Soumia Bouzahar,
}

Université Med Kheider- Biskra, Algérie.

\section{Résumé}

Cette réflexion questionne les récits de voyage et les descriptions des auteurs sur le paysage oasien, un patrimoine actuellement en péril qu'il faudra bientot sanctuariser, afin de comprendre ce que signfie un paysage oasien aux yeux d'un visiteur et de savoir quels sont les éléments visuels et sensoriels qui définissent sa representation mentale.

Cette passionnante rechreche a mis sous la lumière une "relation d'échange de service écologique" entre le visiteur et l'agrosystème oasien. Cette relation illustre approximativement la vision d'un écutouriste ou plus simplement un touriste conscient de la fragilité de ce paysge.

Mots-clés: Ziban, éléments visuels et sensoriels, paysage oasien, relation d'échange de service écologique, les récits de voyage.

\section{Définition du cas d'étude «Biskra la micro région des Ziban »}

Située au sud est de l'Algérie (Fig. 1), au pied sud de la chaîne montagneuse de l'atlas saharien qui représente les monts des Aurès ainsi que la limite entre le nord et le sud algérien, produisant ainsi un authentique espace tampon. Cette situation lui a value la connotation de " porte du désert » et lui a permis de jouer à travers les différentes époques de son existence un rôle de lieu de rencontre et d'échanges. La microrégion des Ziban constitue espace de transition entre un nord du pays bien équipé et un sud déshérité. Mais, 
elle reste l'un des espaces les plus attrayant et le plus fragiles dans le monde par sa structure paysagère, ses vues panoramiques et sa simplicité.
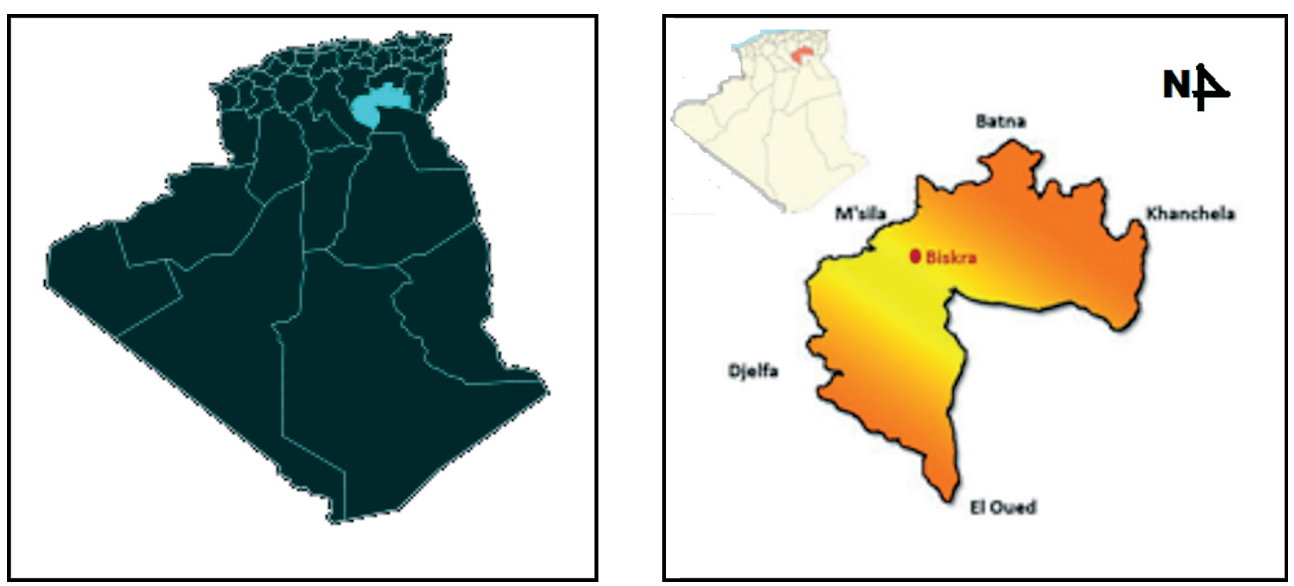

Fig. 1 - La situation de Biskra en Algérie. Source: (ANDI, 2013)

\section{1. Composantes géographique du paysage oasien Zibanais}

La wilaya est située au sud - est de l'Algérie au nord du bas Sahara. Avec une altitude de $112 \mathrm{~m}$ au niveau de la mer. Ce qui fait d'elle une des villes les plus basses d'Algérie. Le Chef lieu de la wilaya est située à $400 \mathrm{~km}$ au Sudest de la capitale, Alger. La wilaya s'étend sur une superficie de 21671 km². (ANDI, 2013)

Le relief de la micro région des Ziban se divise en quatre grands ensembles. On trouve une chaîne montagneuse, les hauts plateaux au nord et les oueds; avec la plus grade l'altitude de djebel Tekriout $1942 \mathrm{~m}$. Puis : (monographie de la wilaya, 2011)

- La montagne d'Elkaid

- La montagne de H'mara

- La montagne de Guessoum $1087 \mathrm{~m}$

- La montagne de $\mathrm{R}^{\prime}$ baa $712 \mathrm{~m}$

- La montagne de Kara

- La montagne de Bourezal

- La montagne d'Amlili $1496 \mathrm{~m}$

- La montagne de Houdja 1070m

- La montagne d'Ahmar Khadou 
La plus part de ces montagnes sont désertes et sans couverture végétale naturelle. (Direction des forêts, 2011).

Les grands plateaux se trouvent au sud ouest de la région notamment sur la région de Ouled Djellal ; et Sidi Khaled.

Les plaines steppiques s'étendent à l'est, sur l'axe El-Loutaya et Tloga pour englober les plaines de Sidi Okba et Zribet Eloued.

Les dépressions situées au sud-est caractérisées en une surface lisse d'argile qui contient des couches minces d'eau qui représentent les chotts dont le plus important le chot de Melghighe. La baisse moyenne (- $33 \mathrm{~m})$ en dessous du niveau de la mer, est donc le composé principal de l'eau de surface naturelle dans la région. (Direction des forêts, 2011) (Fig. 2).

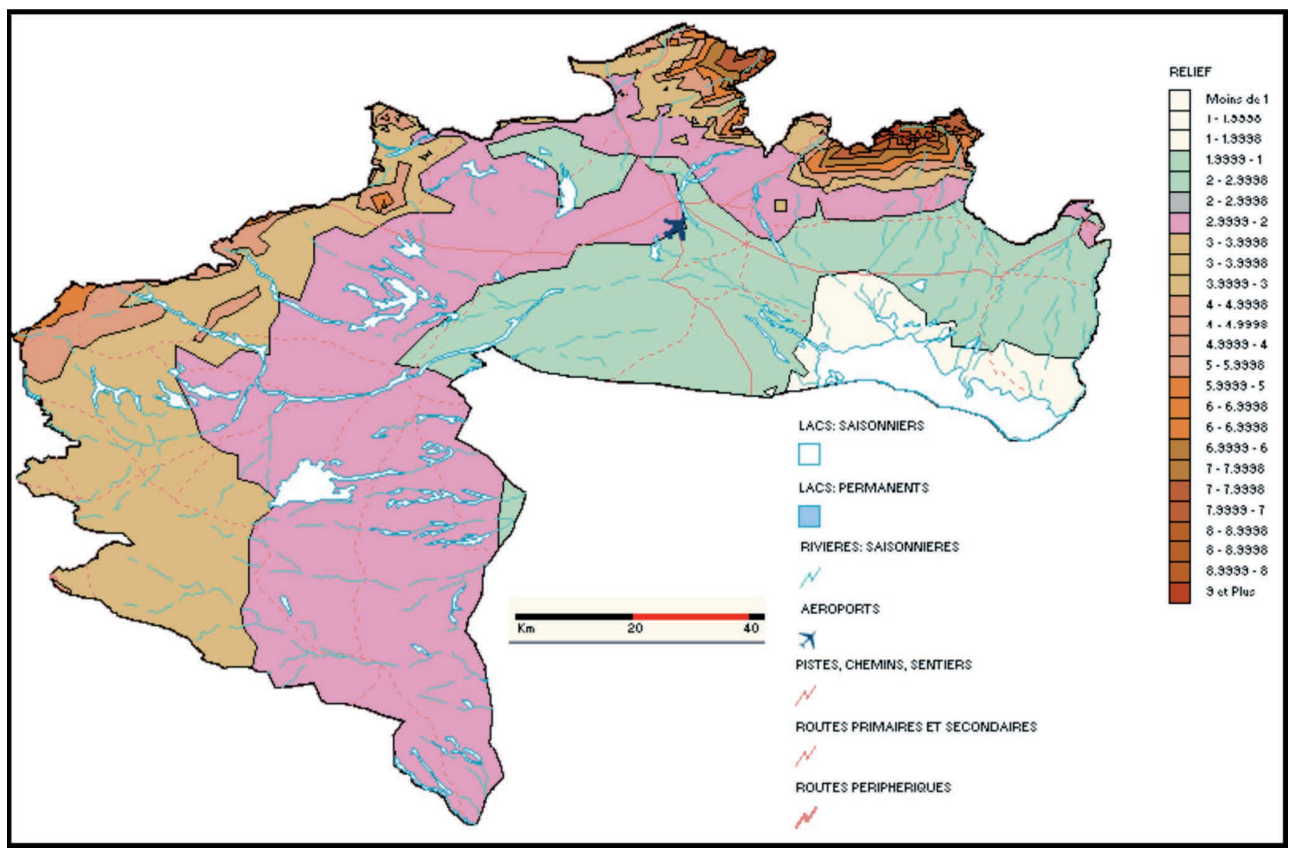

Fig. 2 - La répartition du relief, les réseaux hydrauliques de la micro région des Ziban. Source : Health-Mapper ver 4.1

\subsection{Réseau hydrographique}

Il est constitué par des oueds suivants.

- Les oueds des Aurès : Ils tirent leurs origines du cœur des Aurès, contenant de grands bassins, y compris:

- Oued Elhai et oued Abdi, qui compose oued de Biskra lors de leur 
rencontre.

- Oued arabe et oued al-Guattan, qui compose oued Zriba lors de leur rencontre à Zribet Eloued.

Le débit de l'eau dans ces oueds est minime en hiver et sec depuis le début du mois d'Avril.

- Les oueds du pied sud des montagnes des Aurès :

Caractérisé par ses petits bassins, ce qui rend la course d'eau, peu et irrégulière.

- Les Oueds du Zab est n'atteint pas le chot, sauf dans le cas d'une inondation.

- Les Oueds d'Ouled Djelal versent principalement dans l'oued Djdaia.

- Les Oueds d'Eloutaya contribuant ainsi à recharge des eaux souterraines par le biais de son absorption par le sol.

- Oued Djdaia :

Un Bassin de $26000 \mathrm{~km} 2$ et une longueur de $500 \mathrm{~km}$ c'est le collecteur principal et naturel de toutes les eaux de l'Atlas saharien. Comme d'autres Oueds désertiques la plupart du temps il est sec, il n'est rempli qu'en période d'inondation. (Direction des forêts, 2011).

\section{1. 2. Composante climatique du paysage oasien Zibanais}

Par sa situation elle constitue un carrefour important de lien entre les villes du nord particulièrement celles de l'est et les villes du sud. Cette implantation lui a confié un climat désertique rigoureux, sec et aride, dont des hivers froids et secs et des étés chauds et secs ; la température moyenne annuelle est de 21.8 ${ }^{\circ} \mathrm{C}$, avec un minimum de $3,4^{\circ} \mathrm{C}$ en janvier et décembre et une température un maximale de $49,5^{\circ} \mathrm{C}$ en juillet et août. (Direction du transport, 2011) Quand aux Précipitations la station météorologique de Biskra, au cours de la période 1967-2005 a enregistré $136 \mathrm{~mm}$ /an de précipitation moyenne dans la région pendant 35 jours ; considérée comme très faible. (Monographie de la wilaya, 2011)

La région se caractérise d'une solarisation moyenne annuelle d'un ordre de 3417 heures, ce qui la rend très ensoleillées. Le mois de juillet culmine 383 heures, par contre le mois de novembre avec 210 heures du soleil. La rose annuelle des vents illustre la prédominance de deux courants d'air respectivement des secteurs nord-ouest et sud-est. En générale Les vents 
apparaissent lors des changements des saisons. Les vents de sable viennent frapper la région à la longueur de l'année et surtout en mois d'avril, mai et juin dont ils atteignent le nombre de jours le plus élevé, le sirocco qui est particulièrement fort et chargé généralement de sable vient du sud du mois d'avril au mois d'octobre, il atteint son max le mois de juillet ou il enregistre une moyenne de 20 jours.

\begin{tabular}{|l|l|l|l|l|l|l|l|l|l|l|l|l|}
\hline Mois & Jan. & Feb. & Mar. & Avr. & Mai & Juin & Juil. & Aout & Sept. & Oct. & Nov. & Dec. \\
\hline T. $\max ^{\circ} \mathrm{C}$ & 16.1 & 18 & 22.4 & 26.8 & 32.2 & 37.2 & 40.6 & 39.5 & 34.4 & 28 & 21.3 & 17 \\
\hline T. $\min ^{\circ} \mathrm{C}$ & 5.8 & 7.6 & 10.2 & 13.8 & 18.2 & 23.7 & 26.6 & 26.4 & 22.5 & 16.4 & 11 & 7 \\
\hline
\end{tabular}

Tab. 1 - Prévisions températures mensuelles de Biskra (2016)

Source : Relevées de la station météorologique de Biskra

Cependant ces caractéristiques climatiques et géographiques ont poussé l'homme des Ziban à produire une variété d'établissements humains. Ainsi que plusieurs manières ingénieuses d'adaptation et d'intégration dans les sites naturels, ainsi que plusieurs façons d'exploitation durable des ressources naturelles de ces sites tel : l'eau, l'agriculture, la phoeniciculture pour créer enfin une variété d'oasis sans pareille. Pour mieux comprendre et cerner la micro région des Ziban un rappel historique nous apparaît important et ferra le sujet du sous titre suivant.

\section{La constitution historique du paysage oasien Zibanais}

Biskra, Sokkra, Vescra, Vecera, Adbesran,... toutes ces nominations, ont fait l'objet d'une grande recherche approfondie. Mais les historiens arabes et étrangers ne sont pas en accord sur les origines de son appellation.

\section{1 Chez les romains: rôle des éléments, ponctuel et linaire}

Parmi eux, plusieurs disent que son nom descend du nom romain «VECERA», qui signifie «station» ou «endroit» d'échange commercial, vue sa situation géographique qui relie le nord au sud. Mais le chef romain «BETOLIMIH BENYOUBAII » lui donna un autre nom «la rivière du destin», qui est du à la rivière de sidi Zarzour (le nom de la rivière aujourd'hui) qui travers la ville. Certains chercheurs trouvent que son nom descend de l'ancien nom romain «ADEBESRAN» du à l sancienne source géothermale qui se trouve proche de la ville, «HAMMAM SALHINE» aujourd hui.

Les invasions romaines, aboutirent à une implantation militaire, culturelle et 
religieuse, permettant l'intégration des éléments visuels linéaires tels le limes, les routes et les systèmes d"irrigations et des éléments visuels ponctuels tel les bâtisses et les constructions.

«L'existence des bâtis romains au lieu dit «Khroub» (ruine) située au nord ouest du Besbès ou «Ghebara» à Badès, et « le moulin romain» à Chetma, «le barrage Foum El Gharza» à M'llili ainsi que la présence « d'une forteresse» située dans la commune d'Ouled Djellal indiquent le passage et l'installation des romains dans cette région. »

$\mathrm{D}^{\prime}$ autres sources disent que le vrai nom descend du mot «Sokkra», une description donnée par l'historien IBN KHALDOUN due à la meilleure qualité des dattes sucrées que cette ville possède partout dans ses oasis.

\section{2. Les musulmans et la ponctuation religieuse et fonctionnelle du paysage}

$\mathrm{Au}$ septième siècle, la conquête musulmane commença sous la conduite d'Okba Ibn Nafaa El Fihri, dont le tombeau et le mausolée se trouvent dans la ville qui porte son nom (Sidi Okba) située a18 km à l'est du chef lieu de la wilaya de Biskra dans le Zab Echergui (Oasis Est).

A partir du VIIIe et jusqu'au XIIIe siècle, quatre états se succédèrent dans le territoire: les royaumes Rostemide - Ziride - Hammadite et Wahabide. Chacun d'eux, avait une capitale, située à cheval sur le Tell et les hautes plaines (Cote M.,1988), permettant ainsi des échanges avec le Nord comme avec le Sud, devenant un pôle d'échange et de commerce Transsaharien, sur ce que l'on appelait la route de l'or (or, ivoire, sel et épices). Ce grand axe d'échanges a été le facteur principal de la multiplication des implantations humaines et des oasis dans la zone et l'armature du commerce nomade au point ou selon les récits d'Ibn Khaldoun certaines caravanes comptaient jusqu'à 12000 chameaux.

\section{L'agrosystème, les groupements oasiens Zibanais et le façonnement écologique du paysage}

Sous ces occupations les Ziban ont gardé leur organisation spécifique et bien adaptée à la géographie et au climat de la région. Les Ziban, représentaient un réseau oasien, qu'Ibn Khaldoun a défini dans la «Mokadima» comme: " une grande nation qui se compose de plusieurs villages ». Les Ziban s'organisaient sous la forme de groupements villageois discontinus, le long des oueds. Ceux-ci correspondaient globalement aux terres cultivables et aux palmeraies. (Alkama Dj., 2006) 
Les réseaux villageois des Ziban sont organisés en des entités distinctes, appelée Zab. La différence entre ces Zab est d'abord la qualité des terres, puis les caractéristiques sociales et morphologiques de leurs habitants. (Bouzahar L. S., 2008)

Les citations sont diverses en matières de nombre de Zab qui composent la microrégion, certains récits racontent qu'il y'a 02 Zab Chergui et Gharbi (Alkama Dj., 2006) d'autres disent que Biskra est un Zab aussi, le colonialisme français cite quatre Zab, zab de Biskra Zab Chergui, Zab Dahraoui (sud) et Zab Guebli (nord). (Colonel Niox, 1890).

Les $\mathrm{Zab}$ sont un ensemble de petites oasis regroupées le long des oueds dont chaque oasis est indépendante économiquement et politiquement. Chaque oasis a son propre patrimoine culturel et architectural et ses propres dispositifs et organisations spatiales. (Bouzahar L. S., 2010)

\section{1. L'oasis de Biskra}

«La perle du désert (Ad Piscinam des Romains), est le centre et comme une capitale des oasis des Ziban. C'est le siège du commandement militaire des nomades du bassin du chott Melghir. Riante petite ville, avec de beaux jardins bien arrosés, sous un ciel toujours pur, c'est une des résidences d'hiver les plus agréables de l'Algérie. Aussi la douceur de son climat y attire de nombreux touristes; des villas de plaisance ont même été créées et y sont entretenues à grands frais, mais l'été y est brulant pendant les mois de juillet à septembre. Les nomades, qui campent très nombreux dans ses environs, remontent alors sur les hauts plateaux, et ils n'y restent plus que la garnison française, les agents» (Colonel Niox, 1890).

\section{2. Le Zab Chergui}

L'ensemble du Zab Chergui comprend le territoire situé entre les pentes méridionales de l'Aurès et le chott Melghir, à l'est de l'oued Biskra. Il est caractérisé par une nature agricole plus qu'oasienne. (Colonel Niox, 1890). A l'Est est le lieu de la confrérie Rahmania connu comme des relais $\mathrm{du}$ chemin de pèlerinage. Ces deux petits centres tirent leurs origines de l'époque romaine comme le précise Ibn Khaldoun dans La Mokadima. Le Zab Chergui s'apparente avec des terres cultivables mais une accumulation du capital durablement lié au commerce transsaharien, cette fois-ci en lien avec l'est et le Souf. (Alkama Dj., 2006) 


\section{3. Le Zab Guebli axe sud}

Les oasis sont toutes situées dans la vallée de l'oued Djedi, dans laquelle, au-dessous des sables, on trouve un courant d'eau excellente ce qui reflète son caractère oasien. (Colonel Niox, 1890). Cela constitue une entité considérablement peuplée. Ses terres gypseuses ont donné naissance à de grandes palmeraies qui s'étalent tout le long du pied des montagnes des Amours à l'ouest de la micro capitale des Ziban « Biskra ».

L'ensemble des ces villages oasis, est fondé sur les vestiges de petits établissements humains romains. Nous citons à titre d'exemple : la petite bourgade Bouchegroune, elle est fondée sur un site romain qui s'appelait Ghrada. Lichana était aussi un camp militaire romain entouré par une enceinte en pierre. (Alkama Dj., 2006)

\section{4. Le Zab Guebli axe nord}

Il est séparé du Zab Guebli par une bande de sables et de marécages. Ses oasis sont alimentées par des sources abondantes descendant du djebel Matraf, que l'on traverse en venant d'el-Outaya. (Colonel Niox, 1890).

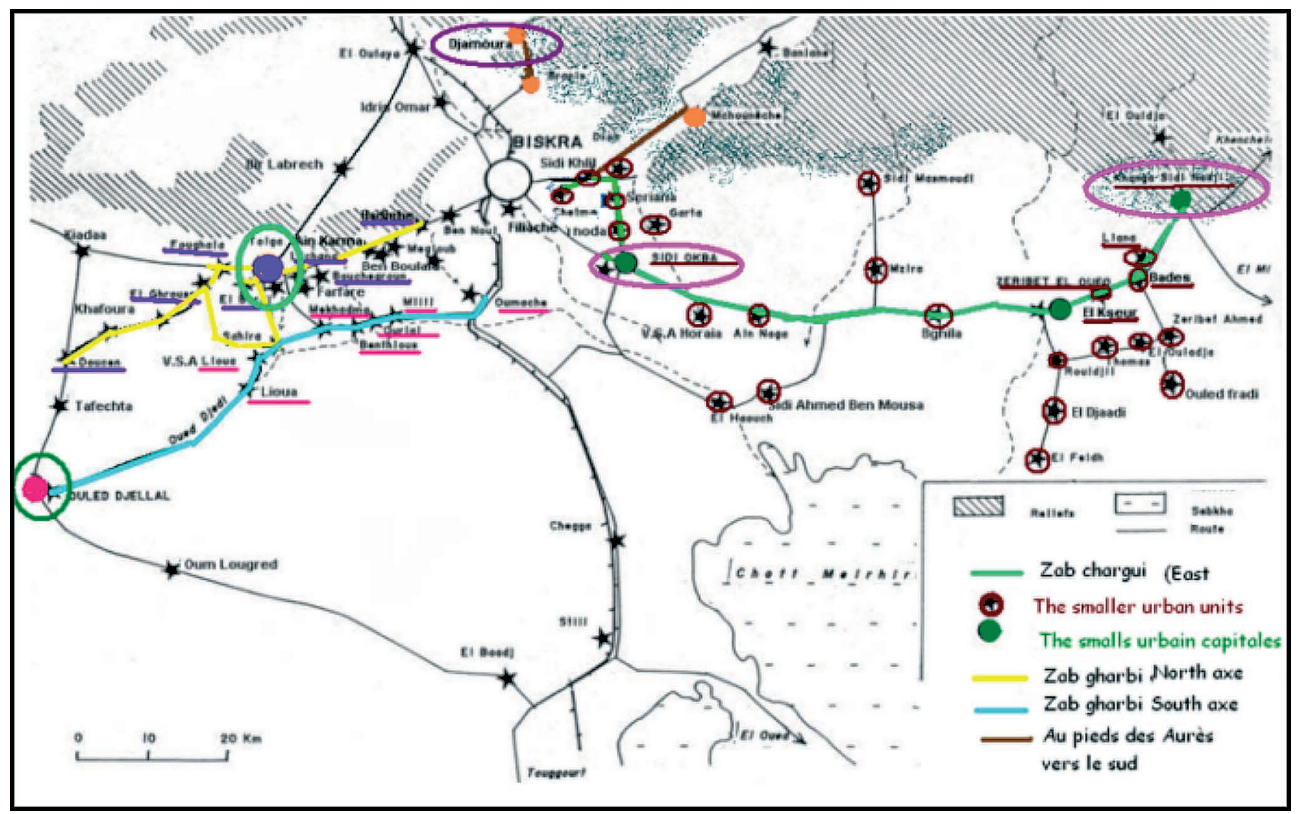

Fig. 3 - Les plus petites unités urbaines et leurs Capitales pour chaque Zab Source : Auteur \& Pf. Alkama(2008) 


\section{5. Le groupement des bas plateaux des Aurès}

Djamourah, Branis, Guedila, Beni Souik et Elkantara sont les établissements humains des bas plateaux des Aurès, ils sont déterminés en plus du nombre réduit en palmiers par les arbres fruitiers. (Colonel Niox, 1890).

Au pied de la montagne du versant sud des Aurès se succède un ensemble d'oasis sans pareil. Il s'agit de : El Kantara, Djamoura, Guedila, Béni Souik, Ain Zaâtout et M'chounech.

\section{Des groupements oasiens qui respectent durablement les paysages}

Une forme urbaine durable résumée dans l'article 1 de la déclaration de Rio «Les êtres humains sont au centre des préoccupations relatives au développement durable. Ils ont droit à une vie saine et productive en harmonie avec la nature.» Ces fondements sont :

- Le bien être de l'homme dans son contexte urbain, social et environnemental ; tout en cherchant une cohésion sociale et solidaire entre les territoires et entre les générations, incontournable du progrès social.

- L'équilibre écologique entre le cadre de vie de l'être humain qui constitue 'l'écosystème urbain' et l'environnement naturel au quel ils appartiennent et qui est 'l'écosystème naturel' dans le souci de la préservation de la biodiversité.

- Un projet qui garantisse un développement local, suivant des modes de production et de consommation responsables, c'est-à-dire à la fois moins polluants et moins dégradants, moins prédateurs en termes de ressources et de milieux naturels, moins consommateurs des terres, et limitant au maximum les risques pour l'environnement et les conditions de vie sur terre.

- Finalement la notion participative entre tous les acteurs, les agents, les habitants de cette forme urbaine.

En guise de synthèse à ces fondements, il s'agit de comprendre, de préserver et de maintenir la trilogie : cité, citoyen, et oasis. L'intérêt de ce titre et d'étudier cette trilogie et ces fondements dans les Ziban.

\section{1. Des poches urbaines de densité moindre noyés dans la palmeraie}

La ville durable repose sur le respect de l'environnement en tenant compte des caractéristiques climatiques et en s'orientant vers des matériaux 
respectueux de cet environnement. Et quand la durabilité vise à, préserver le capital économique, social et naturel de manière à répondre aux besoins du présent, sans compromettre la possibilité pour les générations futures de satisfaire les leurs, grâce à leurs palmeraies les groupements humains des Ziban seront la parfaite illustration. (Bouzahar S., 2009).

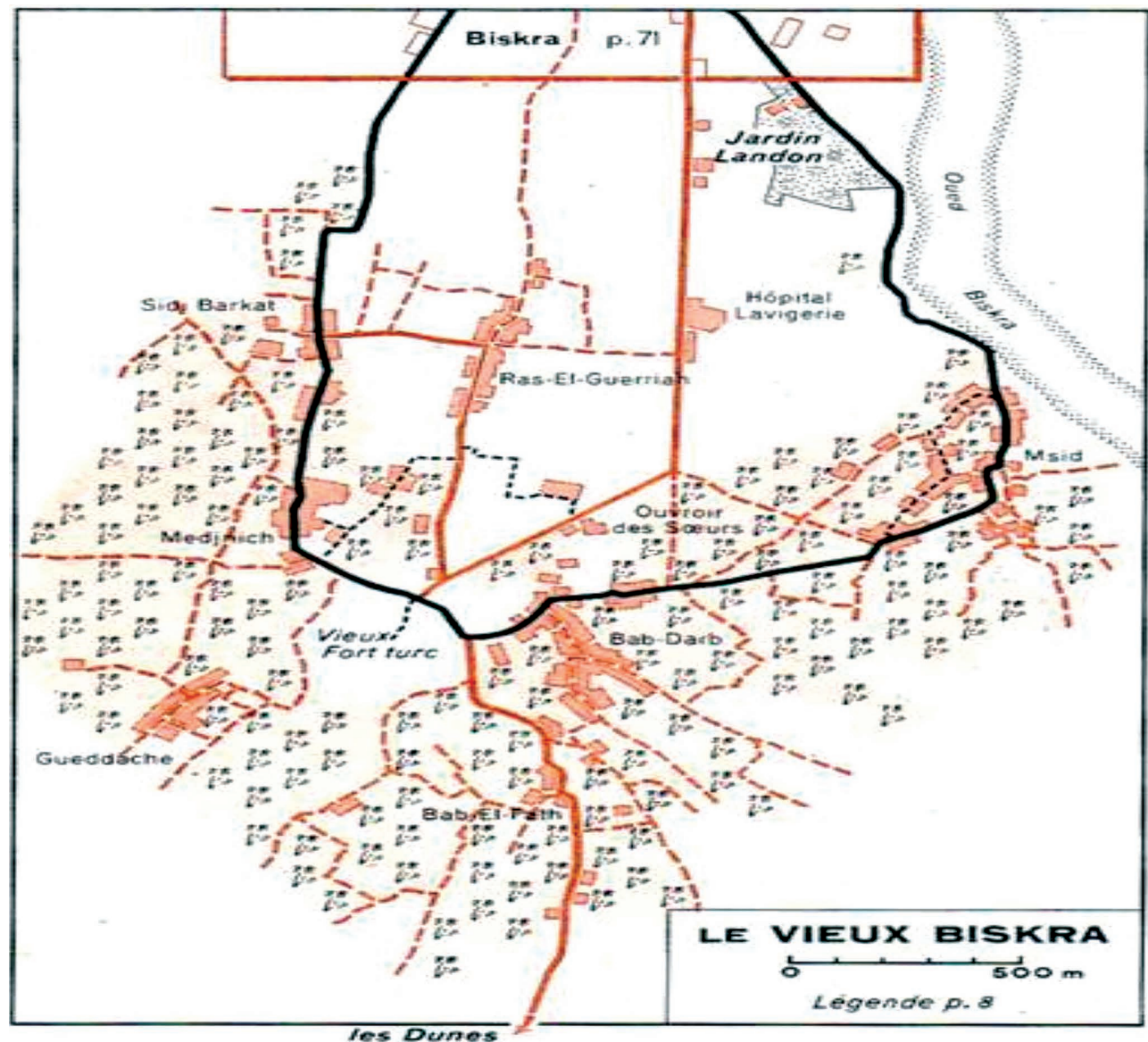

Fig. 4 - Les premiers groupements humains du Zab de Biskra noyautés dans leur palmeraie Source : Carte Michelin n 172- plis 17 et 18

Le Vieux Biskra compte l'ensemble des ksour qui groupent leurs maisons aux murailles croulantes dans la célèbre palmeraie de 150.000 palmiers. Les villages disséminés dans l'oasis sont bâtis en "toube " (brique de terre et de paille foulée et séchée au soleil). La meilleure façon de visiter en auto le 
Vieux Biskra et de parcourir les parties les plus intéressantes de sa palmeraie et de suivre l'itinéraire indiqué sur la carte de la figure 4. (Biskra guide vert)

\subsection{Le respect de services écologiques à l'échelle du plan de masse}

Les Ziban représentaient une des meilleures formes urbaines durables dans les régions oasiennes à écosystème fragile, par les échanges de services écologiques avec leur environnement. (Bouzahar S., 2008) La surface de l'établissement humain ne dépasse jamais la surface du palmier ou des terres cultivables. (Bouzahar S., 2011) Le palmier comme élément naturel révèle au-delà de ses aspects économiques et techniques, des critères écologiques et sanitaires. (Wackermann G. et all, 2005)
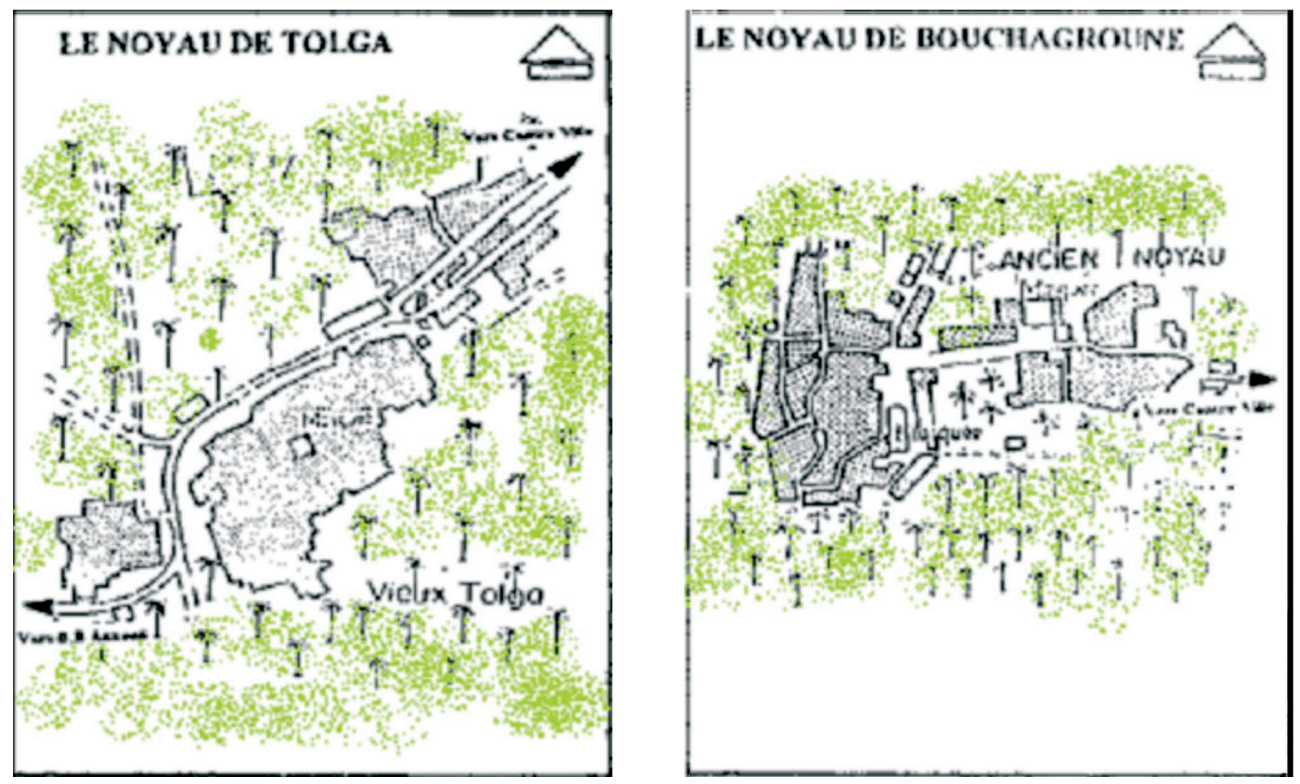

Fig. 5 - Les unités urbaines (a) Tolga. (b)Bouchagroune noyautées dans leur palmeraie. Source : Alkama Dj., 2010

\section{1. 2. Le respect des services écologiques population/établissement}

À chaque fois que le nombre de population augmente, il y a lieu de construire un nouvelétablissement humain, en gardant un lien social avecl'ancienétablissement et d'autres liens d'ordre fonctionnel ou religieux tel la mosquée, le sanctuaire. L'exemple de Farfar à Tolga ou bien le cimetière à de Ghoufi. (Bouzahar S., 2011) 


\section{1. 3. Le respect des services écologiques population/terres agraires}

Chaque fois que le nombre de population augmente la surface des terres cultivables augmente aussi étant donné que c'est leurs sources de vie. Ce qui produit un cadre de vie social, artificiel (cadre bâti) et naturel, écologiquement équilibré.

\section{2. Les projets économiques et le façonnement durable du paysage oasien}

Les aires de palmeraie ont favorisé la création de petites unités urbaines comme elles ont favorisé une auto-soutenable de chaque unité urbaine. Elles se sont insérées dans le paysage existant, comme elles ont façonné écologiquement le paysage sans le détruire.

Chaque Zab avait son propre projet économique, l'oasis de Biskra était spécialisée dans le commerce et les échanges vu sa situation centralisée géographiquement et à l'intersection des autres Zab.

\section{2. 1. Le Zab chergui}

Était spécialisé dans la production des céréales: Badès, l'ksar et Liana étaient spécialisés surtout dans la production du blé et de l'orge. Zribet Eloued était spécialisé dans l'agriculture des plantes médicinales et des herbes (henné, cumin, safran, rosier ...), jusqu'au tabac et des cultures maraîchères (haricots, oignons, carottes, piments, tomates, pommes de terre ...). Les deux capitales de ce Zab Sidi Okba et Khanguet Sidi Nadji étaient spécialisés dans la phoeniciculture et dans les cultures sous étagées.

«Sidi-Okba : ville sainte de l'Islam magrebin. $34 \mathrm{~km}$ en auto AR plus $1 \mathrm{~h}$. de visite. Quitter Biskra par la rue Lazerge et la sortie $n^{\circ} 2 \mathrm{du}$ plan. Après avoir traversé l'oued Biskra, prendre à droite une piste goudronnée qui traverse les petites oasis d'El-Alia, et de Filiache, puis parcourt pendant $15 \mathrm{~km} \mathrm{le}$ désert sablonneux en vue du massif de l'Aurès, à gauche, avant d'atteindre la palmeraie et la ville de Sidi-Okba. » (Biskra guide vert) Sidi Okba contenait 66.000 palmiers, célèbre par la mosquée où est enterré le premier conquérant arabe.

Khanga-Sidi-Nadji, oasis de 28.000 palmiers produisant des dattes très renommées où la culture des céréales est aussi pratiquée. Un barrage est établi en avant de Khanga ; il retient l'eau pour l'irrigation de l'oasis et des terres de labours, les 02 tiers des eaux de l'oued El Arab . 
Zéribet El Oued (le clos de la rivière) a 1.100 habitants avec un petit fortin ; ses palmiers au nombre de 1.200 seulement sont disséminés au sud et sur la rive gauche de l'oued El Arab où ils abritent la Kouba de Sidi Hassen El Koufi, arabe de l'Hedjaz qui aurait jadis fait couler miraculeusement l'eau de la rivière. De Liana à Zéribet El Oued le terrain est parsemé de cailloux. L'oasis de Zéribet El Oued a des terres de culture d'une certaine importance ; elle est dotée de 70 puits donnant un débit de $7.000 \mathrm{~m} 3$ d'eau par 24 heures.

\section{2. 2. Le Zab guebli}

Lui était spécialisé dans la production des dattes, les meilleures dattes au monde. D'ailleurs sur les plans il apparait comme une barrière écologique oasienne. Chapoté par l'oasis de Tolga.

Tolga : C'est la plus importante oasis des Ziban. Ses rues très animées offrent un spectacle pittoresque. Sa mosquée est intéressante. Du haut de son minaret, auquel on accède par un escalier s'élevant autour d'un massif pilier carré, on jouit d'une vue d'ensemble sur l'oasis. Sa palmeraie abrite de vastes jardins arrosés par l'eau de puits artésiens dont on voit les larges conduits modernes. (Biskra guide vert)

Lichana : A 1,5 km de la piste. Vaste ksar pittoresque, la palmeraie alentour produit d'excellentes dattes. (Biskra guide vert) Farfar. : Village pittoresque et coloré. Sa palmeraie verdoyante s'étend au bord de la route. (Biskra guide vert)

\section{2. 3. Les oasis des bas plateaux des Aurès}

Leurs productions agraires constituées d'arbres fruitiers variées tels : prunes, abricots, figues, pêches, olives... etc. Parmi ces oasis nous citons. L'oasis de Djemorah où les Ouled Ziane s'installèrent au début de leur arrivée dans l'Aurès; les Ouled Ziane rayonnèrent sur les autres tribus. Le sol de cette oasis formé d'alluvions sur les bords de l'oued Abdi est escarpé et composé de schistes à bancs successifs de carbonate de chaux dans les autres parties. Les calcaires dominent encore dans les rochers qui encaissent l'oasis. Le palmier forme la culture principale du pays on en compte 06 qualités qui sont par ordre de mérite : le deghlat-nour, le kertiche-deghlat, le kertiche, el-djaouzi, al-ghars et el-zerrat. Le miel de Djemorah est très renommé et se vend bien.

L'oasis de Djemorah appréhendait 80.000 palmiers; elle s'étend sur 
plusieurs kilomètres de parcours, elle est arrosée par 08 prises d'eau faites sur l'oued Abdi et par de nombreuses sources très abondantes contenues dans l'oasis même (elles sont au nombre de 18).

Guedila (ou Keddila) à $5 \mathrm{~km}$ de Djemorah. Sur les flancs de coteaux, située à droite de la route de Djemorah à Biskra, on trouve la petite oasis de Guedila, située en dehors du cours de l'oued Abdi; elle est irriguée par des sources assez abondantes et très poissonneuses (les indigènes racontent que les sources ont tari à un certain moment parce qu'on $y$ avait pris du poisson. Aussi est-il formellement interdit d'y pêcher). Branis ou Branès: oasis de montagne. $44 \mathrm{~km}$ en auto AR plus $1 / 2 \mathrm{~h}$. de visite. Sa route s'élève d'abord vers le col de Sfa d'où se révèle un large panorama sur le désert au Sud. Au pied du col de Sfa, sur le versant Nord, ... on pénètre dans la montagne et la route atteint les premiers contreforts de l'Aurès. ... l'oasis de Branis apparaît dans son cadre de montagnes très colorées. Sa palmeraie s'étend, bien irriguée, dans la vallée de l'oued qui décrit là un méandre à l'intérieur duquel s'élève le village. (Biskra guide vert)

Elle contenait 20.000 palmiers. Elle utilise une grande partie de ses eaux, le reste se rend dans les plaines d'El Outaya au lieu dit «Dar Arous» ou «Dar El Aroussa».

La réussite économique de cette organisation est due à l'exploitation de l'agrosystème. Il s'agit d'un écosystème oasien créé par l'homme des Ziban afin de subvenir à ses besoins, alimentaires notamment. Dans cet agrosystème, $l^{\prime}$ homme favorise le palmier au dépend des autres plantes.

- Une identité rurale oasienne distinctive

- Une société rurale particulière

- Une répartition urbaine respectueuse de l'environnement.

- Une architecture spécifique (matériaux respect de l'environnement).

- Un rapport entre terres cultivées, palmiers, bâti et population.

\section{La situation du paysage oasien face au colonialisme français}

En 1837 l'occupation française entreprit la conquête de la zone sud est ; en 1844 la région est officiellement intégrée sous administration coloniale. 5.1. Les premières désorganisations structurelles : Plusieurs dispositifs et mesures étaient pris pour le contrôle et l'exploitation de la colonie conduisant ainsi à plusieurs effets non désirable sur la société ou sur l'environnement. 


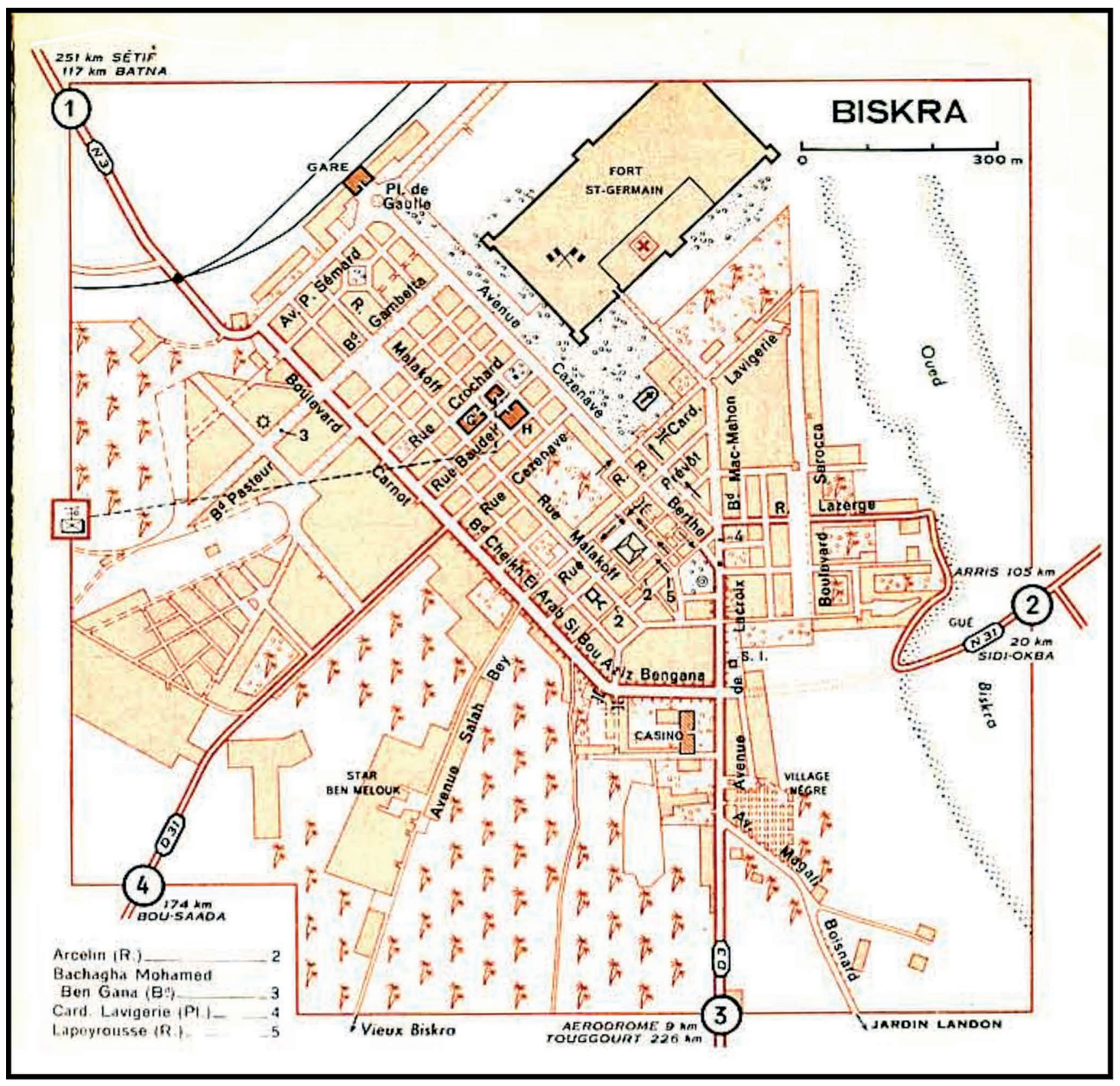

Fig. 6 - Les prémices de la modification du paysage par le colonialisme français. Source : Cardinal Lavigerie, 1956.

\section{1. 1. Des mesures discriminatoires et privatisation des terres tribales}

Une série de mesures et de décisions politiques et administratives souvent discriminatoires sont prises par l'autorité coloniale, comme par exemple l'assignation de la population à son origine ethnique et religieuse en vue de mieux contrôler la population musulmane. Le fait d'appartenir à une région différente et non intégrée au code civil français (code de l'indigénat) impliquait l'exil et la marginalisation. Ces décisions visaient à privatiser et à 
récupérer les terres tribales, surtout les meilleures terres au profit des colons. Cet état de fait aboutit à la désorganisation des structures communautaires séculaires aux profits d'un système colonial exogène "foncièrement organisé ». L'administration coloniale instaure des implantations agricoles productives selon de nouvelles méthodes et valeurs qui sont contraires aux anciens dispositifs de solidarités à travers la gestion collective des terres agricoles par les Arch. Les entraides lors des récoltes par les systèmes de Touiza, ont divisé les membres appartenant à la même famille ou le même clan.

\section{1. 2. Les infrastructures hydrauliques et les réseaux routiers}

Pour faciliter l'exploitation des terres et les échanges commerciaux avec le Nord notamment pour les produits animaux, les dattes, les fleurs, le tourisme..., des infrastructures hydrauliques ainsi que des réseaux routiers, ferroviaires et électriques sont réalisés.

A partir de 1947, l'administration coloniale réalise plusieurs infrastructures à Biskra parmi lesquelles une retenue collinaire avec une seguia $(10 \mathrm{Kms})$ sur l'Oued Djedi, pour irriguer la palmeraie, ainsi qu'un forage albien avec un système de refroidissement pour irriguer la palmeraie de Sidi Khaled. Besbes et sa région bénéficient d'un puits d'abreuvement à Dendougui 1956, Besbes1956, Fehama 1957, El Goutaa 1958. Des djoubs sont par ailleurs réalisés à partir de 1952 à travers les oueds pour l'abreuvement du cheptel.

Les parcours n'ont pas été confisqués par l'administration coloniale, cependant elle exerçait un contrôle sur le déplacement des nomades, qui étaient parfois regroupés dans des endroits sous surveillance militaire qui opérait des confiscations de bien ou des impôts aux éleveurs nomades, sur le cheptel Ovins ; Caprins ; camelin et équin.

La colonisation a crée de nombreuses ruptures, entre L'algérien et sa terre, entre ville ancienne et ville nouvelle, entre Islam et culture Occidentale. (M. COTE, 1988)

\section{2. L'exploitation intelligente de l'agrosystème oasien des Ziban}

L'occupation française introduit un nouveau tissu urbain qui a été créé et allait de paire avec une politique d'inégalité sociale et économique. Cela a eu par conséquent, l'apparition d'une ville à l'opposé du vieux Biskra basée sur un tracé parcellaire en damier. Cette ville située au Nord de la 
palmeraie était destinée surtout aux européens.

La structure de cette organisation urbaine sous forme d'îlots réguliers a subit l'empreinte d'une époque peu artistique et la frappe de fondateurs militaires. Elle est caractérisée par la présence du «Fort Sain Germain» pour contrôler les sources d'eau et se détacher physiquement de la ville indigène.

Cette période a connu l'apparition de la rue rectiligne carrossable, de la rue en galerie couverte et des décors d'arabisance faisant référence à une architecture locale. Cet apport urbain a vu que la palmeraie ne représentait que $70 \%$ et ce malgré la réalisation du jardin public, du jardin London et des placettes (monument aux morts, place Ben $\mathrm{M}^{\prime}$ hidi, jardin du 20 Août...).

\section{2. 1. La micro région des Ziban une attraction touristique}

Le caractère pittoresque de la micro région et ses ambiances, lumineuse et thermique ont conduit beaucoup d'européens à la fréquenter. Le Zab de Biskra est devenu l'illustration d'une oasis à destination touristique dans laquelle les européens viennent passer leurs vacances.

En 1932, la ville de Biskra a connu son premier plan d'aménagement, appelé "plan Dervaux" dont l'objectif était de relier la ville européenne à la ville indigène par :

- La réorganisation de la circulation mécanique.

- La création de Biskra comme une véritable attraction touristique :

- grand jardin public, - golf de 18 trous, - station thermale, - grands boulevards, casinos, hôtels....

Ce projet avait pour ambition de transformer la ville de Biskra en paradis pour les touristes. C'est une vaste composition géométrique basée sur des tracés. Pour la première fois l'assainissement de la vieille ville fut pris en considération. (A. Z. Saouli et Al., 1999) Anglais, Suédois, Hollandais, Américains, Espagnols, Italiens, Maltais, Norvégiens, Pétersbourgeois et Allemands illustraient la gamme de touristes, dont la destination était Biskra.

\section{2. 2. L'apport des Récits sur le paysage oasien Zibanais}

De multiples raison ont conduits que ça soit le colonialisme ou les touristes a 
fraiconté les Ziban. Nous avons essayé dans ce qui suit relire quelques récits (l'Abbe Jean Hurabielle, 1899) afin de mettre au clair la perception que les touristes se font des Ziban.

- Les paysages pittoresques oasiens

"Biskra est appelée, à bon droit, la Perle du Désert, et il n'est point de site qui lui soit supérieur. On peut passer un hiver entier à Biskra, en variant chaque jour ses excursions et en voyant, à chaque promenade, des paysages nouveaux. Biskra possède un avantage unique, c'est d'être entourée d'une série d'oasis, toutes pittoresques, toutes pleines de vitalité, toutes ayant leur caractère particulier. Biskra est encore le point de départ des excursions vers le Sud, dans la direction de Touggourt et d'Ouargla. Mais il faut prendre le train et charger son automobile sur un truc. La petite ligne de Biskra à Touggourt est fort curieuse; elle traverse toutes les oasis de l'Oued R'Hir et en exporte les produits. » (Le Général Bonneval, 2003)

\section{- L'atlas}

"L'atlas saharien rassemble dans les monts des Ksour et le Djebel Amour, ses étroits chaînons parallèles qui s'abaissent peu à peu vers l'Est jusqu'à revêtir des allures de plateau accidenté entre Djelfa et Biskra. L'Aurès est le plus caractérisé de ses massifs montagneux.»

- Les vallées

"Les différents modes d'érosion et la variété des roches ont donné aux vallées d'Algérie une grande diversité d'aspects : gorges aux parois verticales taillées par un torrent, vallées larges dans lesquelles ne coule qu'un mince filet d'eau, vallée au fond horizontal, encombré d'alluvions, souvent marécageuse.»

\section{- Les oueds}

"Cours d'eau à régime intermittent, à sec pendant la plus grande partie de l'année, et dont le lit est remblayé de masses de boues séchées et craquelées, de sables et de cailloux roulés qui constituent leurs alluvions, les oueds sont sujets à des crues subites qui se déclenchent à la suite d'orages et qui peuvent faire passer leur débit de quelques litres à plusieurs milliers de mètres cubes d'eau par seconde.»

- Les gorges et le contraste Tell Sahara

"Paris, la France, l'Algérie ne se doutent pas du magnifiques joyau qu'elle possède par delà les gorges d'El Kantara au seuil même du désert. Ces pages, où il aurait désiré faire passer comme une vision de son azur sans tache et de ses solitudes chaudes et lumineuses, lui serviront du moins à payer son tribut de reconnaissance à Biskra pour les jouissances profondes que son ciel lui a procurées et la santé qu'il lui a rendue.» (l'Abbe Jean Hurabielle, 1899,p.6)

"... je fus vraiment impressionné, comme tous ceux qui m'avaient précédé et, 
sans doute ceux qui m'ont suivi, par le contraste violent qui éclate presque, entre le ciel du Tell et le ciel du Sahara, aux pittoresques gorges d'El Kantara." ".... Si bien par les arabes Foum es Sahra, bouche du désert »

"Le célèbre peintre Fromentin n'a pas été insensible à ces beautés; il a traduit son admiration non seulement en toiles magiques, mais en des lignes si précieuses que les Hôteliers s'en font aujourd'hui une réclame.» (l'Abbe Jean Hurabielle, 1899)

\section{- La palmeraie}

"L'hiverneur qui arrive de bonne heure à Biskra, c'est-à-dire vers la fin du mois d'octobre, pourra assister à la cueillette des dattes dans les palmeraies des vieux ksour.»

"Lorsqu'on approche de l'oasis de Biskra par la voie ferrée, le premier coup d'cil est superbe. En contournant le pied des hauteurs où était jadis perchée la forteresse, on voit, aux derniers rayons du soleil couchant, les vertes palmeraies se détacher sur le fond rouge, pierreux ou sablonneux du désert, et l'on admire toute cette nature étrange noyée dans un océan de lumière d'or. Les montagnes lointaines se colorent successivement de rose, de rouge, de violet et d'opale; mais les couleurs de ce tableau désespèrent l'artiste qui essaie de les reproduire.» (l'Abbe Jean Hurabielle, 1899)

\section{- Les vestiges romains}

«Il existe ... des vestiges nombreux de l'occupation romaine. Les ruines les plus remarquables se trouvent ... à $7 \mathrm{~km}$ d'El Kantara; on y reconnait l'ancien Burgum Speculatorum, construit sous Caracalla 211-217, sur ordre de Marcus Valerius Senecio... Il est facile, en parcourant la plaine, de retrouver l'emplacement de l'ancienne ville romaine et d'en reconstituer le plan. Non loin des Ksour, j'ai fait, à dos de mulet, le tour d'un immense camp de César. Il y'a dans cette région, à n'en pas douter, des ruines inexploitées, des secrets enfouis et des surprises réservées aux archéologues de l'avenir.

... au sud de l'oasis on rencontre l'oued djeddi où l'on peut retrouver des ruines d'un pont romain.... Le Ksar d'Oumèche De-ci-delà on peut remarquer des frontons de porte, des seuils, des pierres, des ruines de toute sorte, traces évidentes de la civilisation romaine» (1'Abbe Jean Hurabielle, 1899)

\section{- La chasse}

"... lorsqu'on chasse le mouflon au sud ouest de ces parages,... jusqu'à l'Outaya la route suivant l'oued d'assez près, passe sur des terrains pleins de cailloux roulés et de fossiles, parmi lesquels on voit des oursins, des huitres et des peignes en grandes quantité.»

"A Biskra les chasses, sont une source d'émotions bien plus exquises soit pour les amateurs, soit pour les hiverneurs de longue haleine. Biskra et ses environs 
immédiats ne le sont pas, exceptés au moment de la migration des cailles. Il faut aller chasser à $20 \mathrm{Km}$ au nord à l'est et à l'ouest, si l'on veut trouver les outardes, les lièvres, les perdrix grises, rouges, les canga et les perdrix de Barbarie qui affectionnent le pied des montagnes.

Il y'a beaucoup de colombes à sidi Okba et dans les oasis de l'est; quelques canards viennent s'égarer du coté d'Oumèche, et de rares bécassines dans les dépressions marécageuses et salines du sud ouest de l'oasis...

Pour voir des autruches, il est nécessaire de s'enfoncer à plus de $300 \mathrm{Km}$ dans le sud.

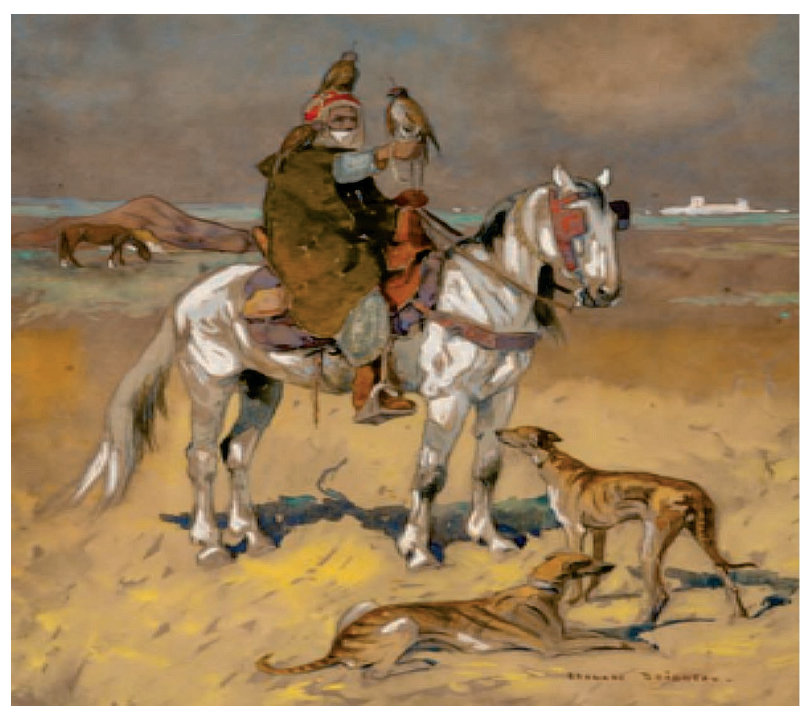

Fig. 7 - Fauconniers aux sloughis. Source : Tableau d'Edouard Doigneau (1865-1954)
Les montagnes environnantes sont particulièrement riches en oiseaux de proie, qui vont chasser dans les plaines : ce sont des aigles, des faucons, des busards, des éperviers, des buses.

On y rencontre d'ailleurs,.... Une grande variété d'oiseaux de passage..., tel que corbeaux, grues, alouettes, étourneaux, merles, grives, chardonnerets, moineaux, hiboux et chouettes... quelques rares ortolans. Le mouflon, ovis ornata El Aroui- se chasse dans les bois de cèdres, de lentisques et de pins d'Alep de l'Aurès, de l'Ahmar Khaddou, de Metlili et du Bent el Arara.

On y trouve aussi l'edmi, antilope ou gazelle des montagnes.

La gazelle commune, autidoracs, des plaines sahariennes erre aux environ d'Ain Naga et quelque fois d'El Outaya. Quant à la gazelle des sables, elle est dans l'extrême sud, comme l'antilope du désert, l'autruche et le guépard.

Il n'est pas rare de voir des hyènes dans les montagnes pelées des environs de Fontaine-chaude. Mais il y'a surtout des chacals dans ces parages... La chasse du renard avec des slouguis dans la montagne est pleine d'intérêt... de la chasse au sanglier dans les monts boisés de Batna, des Chaouia et de l'Ahmar Khaddou septentrional... la chasse aux colombes n'exige pas d'appareil; le passage a lieu 
dans les oasis vers la fin du mois de février et durant le mois de mars. Vers la même époque et aussi un peu plus tard, les cailles remontent du sud vers le nord»

"... les étourneaux s'abattent par nuées sur ces fruits dont ils sont friands, et c'est un amusement pour le chasseur paisible qui n'aime pas les excursions violentes et couteuses, de faire la guerre à ces petits maraudeurs, à la grande joie des felahs qui ouvrent toutes grandes les portes de leurs jardins.» (l'Abbe Jean Hurabielle, 1899)

Ce qui illustre la relation d'échange de service écologique entre les fellahs et les touristes. (Bouzahar L. S. 2015)

\section{- Les courses}

"Elles ont lieu vers la fin du mois de janvier et revêtent un éclat inaccoutumé qui attire un tel nombre de touristes que les hôtels et les maisons particulières ne peuvent loger... Les courses ont lieu dans la petite oasis de Beni Mora, où les tramways de la société de l'oued righ ont une gare en face de l'ancien Tir aux pigeons, dans un parc d'essai abandonné.

Le programme comporte habituellement, le premier jour, une grande course saharienne de mehara, instituée en 1890 par le Cardinal Lavigerie... cette course, si originale, se fait entre Ouargla ou Tuggurt et Biskra : elle est combinée de manière à permettre à celui des compétiteurs qui semble devoir arriver le premier d'être en face de Cora, sur la route de Tuggurt, vers neuf heures du matin après avoir parcourut $336 \mathrm{Km}$. Aussi voitures et cavaliers encombrent ils déjà la route pour assister à l'arrivée triomphale du méhari vainqueur, que l'on escorte ainsi jusqu'à Biskra, après l'avoir magnifiquement pavoisé.

La deuxième journée, des primes sont accordées aux poulains de race arabe, Barbe, ou croisés Barbe-arabe et de Tuggurt; ce concours a lieu dans le jardin public, devant le fort Saint Germain (actuellement jardin El Beilek), dans la matinée. L'après midi ont lieu dix courses avec prix divers, elles sont ordinairement suivies d'une chasse au faucon,

Le troisième jour, après six courses ordinaires, dont une de steeple-chase militaire, ont lieu un défilé et un tour de piste des mehara ayant accompli le trajet Tuggurt-Biskra.»

\section{- L'usage des animaux}

Ils sont le moyen de déplacement et d'investigation agraire et économique. Ils représentent l'anneau articulateur entre être humain et écosystème naturel. Voilà quelque récit de voyage sur le rôle important de la présence de l'animal dans le paysage oasien et agraire :

"Une des routes les plus intéressantes est ...la route des Ziban. On peut la parcourir à bicyclette jusqu'au marabout de Sid Ghezal ; passé ce point, la 
chaussée ne permet plus se genre de locomotion, et force est aux touristes d'aller à cheval ou en voiture..."

\section{- Bourricots et dromadaires}

"La silhouette trottinant $d u$ "bourricots " fait partie de la vie rurale. De petite taille, robuste et docile, toutes les corvées lui sont dévolues. On le rencontre au long des routes et dans les villages, travaillant aux champs ou partant vers les marchés voisins les produits des Ksour et des douars, avançant à pas menus, talonné sans répit par son maître.

Le dromadaire, "vaisseau du désert ", fortune des pays du sud, s'en va roulant et tanguant. Il affronte sans brancher le soleil et la soif. ».

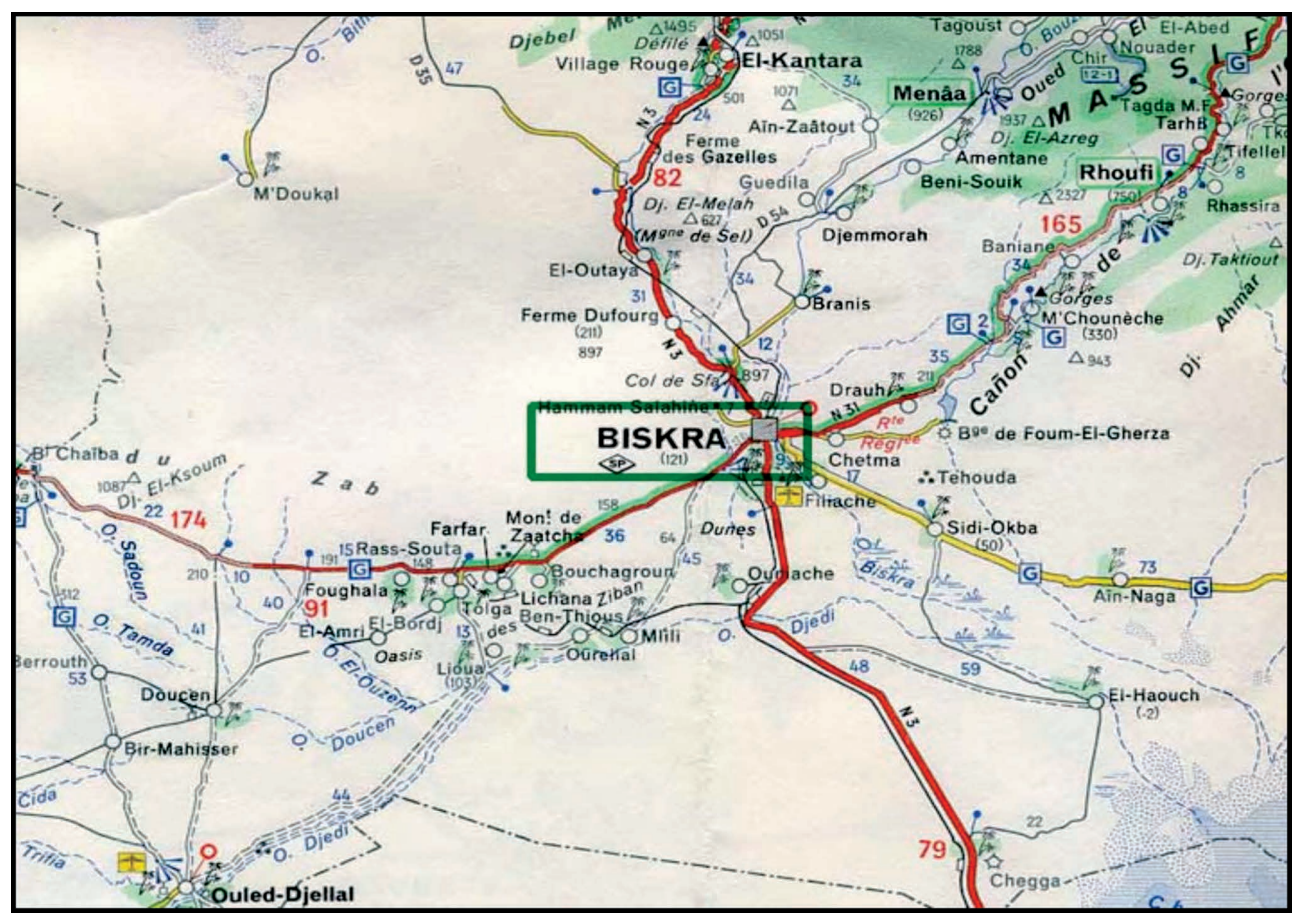

Fig. 8 - Les routes qui desservent les plus grandes oasis des Ziban. Source : carte Michelin, Biskra.

\section{- Le tourisme thérapeutique et les Bains maures}

«... au pied du djebel Khroubs et est situé El Hammam, ou thermes Aquœ Herculis ; une piscine profonde de quatre à cinq pieds reçoit non loin de là les eaux $\left(36^{\circ}\right)$ du Khroubset qui ont une odeur hépatique et une saveur saline prononcée.» 


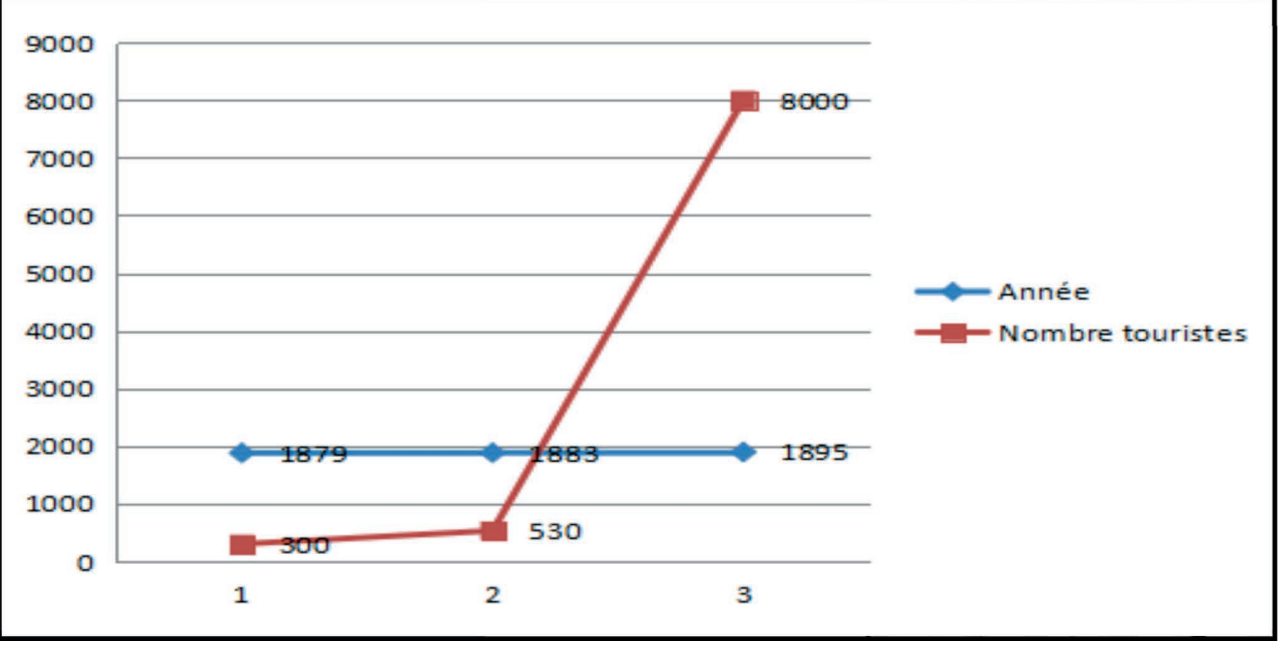

Fig. 9 - Courbe de la croissance de la population touristique. Source : Les données de L'Abbe jean Hurabielle, 1899

"... Depuis longtemps les médecins ont compris quelle était l'excellence de sa situation et de son climat. Aussi s'habituent-ils à diriger plus nombreux chaque hiver les victimes des brumes septentrionales vers ces latitudes ensoleillées. Malades, débilités, simples curieux accourent vers la reine des Ziban se régénérer au soleil du Sahara, ce thaumaturge souverain et incomparable »

"Il y'a une vertu dans le soleil » disait Lamartine en présentant au monde littéraire le chef-d'œuvre de Mistral; c'est à coup sûr, un remède merveilleux et il constitue, aux yeux des thérapeutistes, l'un des agents les plus efficaces qui puisse aider la science pour la guérison radicale de certaines maladies, ou du moins leur soulagement temporaire. Cette prodigieuse quantité de lumière et de chaleur qui brûle les régions désertiques rend, au contraire, la vie et la santé aux victimes des intempéries septentrionales.»

"Cet éden fut de tous les temps l'abri des hiverneurs, le rendez vous familier de ceux qui voulaient échapper aux rigueurs des pays glacés....

Ce qui le prouve, c'est que le Dr Sérizait la signalait en 1865 à l'attention des médecins et des étrangers; ;... les touristes qui visitent l'oasis sont sûrs d'y trouver désormais le genre de confortable le mieux en rapport avec le climat. Que l'on y amène les eaux de Fontaine-chaude, et Biskra deviendra la meilleure station hivernale de l'Algérie» 
Il est bon de comparer, les températures et les états pluviométriques de l'Oasis de Biskra avec ceux de Nice comme une des meilleures stations hivernales.

\begin{tabular}{|l|l|l|l|l|}
\hline & T. max & T. min. & T. moyenne & Pluie \\
\hline Nice & $20^{\circ} 392$ & $2^{\circ} 732$ & $11^{\circ} 412$ & $99 \mathrm{~mm} 495$ \\
\hline Biskra & $21^{\circ} 429$ & $8^{\circ} 914$ & $14^{\circ} 942$ & $17 \mathrm{~mm} \mathrm{425}$ \\
\hline
\end{tabular}

Tab. 2 - Comparaison des températures et de la pluviométrie de deux stations hivernales Biskra/Nice (1899) Source : Les données de L'Abbe jean Hurabielle, 1899

Ce sont là des chiffres qui ont leur éloquence et qui permettent d'opter en connaissance de cause entre l'une et l'autre des deux stations hivernales.

Il résulte des observations ci-dessus que si la température maxima est sensiblement la même, la température minima offre un écart de plus de $6^{\circ}$ à la faveur de Biskra, ce qui en fait, on le comprend, une capitale importante. Un relevé de températures fait par Mr Colombo de 1887 à 1891 soit environ de 5 hivers. Ce relevé explique pourquoi les Ziban se sont vite transformées en la plus importante destination touristique hivernale.

\begin{tabular}{|l|l|c|c|c|c|c|l|}
\hline Mois & Oct. & Nov. & Dec. & Jan & Feb. & Mar & Avr. \\
\hline T. $\max ^{\circ} \mathrm{C}$ & 28,1 & 31,1 & 16,3 & 15,2 & 17,2 & 26 & 26,1 \\
\hline T. $\min ^{\circ} \mathrm{C}$ & 15,2 & 9,2 & 5,7 & 4,4 & 5,8 & 9,4 & 12,7 \\
\hline
\end{tabular}

Tab. 3 - Relevé de températures de 1887 à 1891 Source : Hubert Cataldo, 1988

"Dr. Treille, professeur à la faculté de médecine d'Alger et sénateur à Constantine, s'extasie sur le climat de l'oasis : "Il faut au malades dit-il la vie au grand air, une température douce, par conséquent un air chaud, sec et absolument pur. Tout cela se trouve à Biskra pendant six à sept mois de l'année.»

"Il convient d'observer que cette station permet aux malades d'accomplir une véritable cure hivernale; ils trouveront, en effet, dans ce pays privilégié, non seulement l'air approprié et la température douce, mais aussi les eaux bienfaisantes " dont l'effet, dit le Dr Weisgerber, est plus indiqué en hiver qu'en été pour les rhumatisants, par exemple, qui ne les trouvent concurremment qu'à la station égyptienne d'Hélouan. » Dr Weisgerber avance aussi que "les albuminuriques feront même bien de séjourner plus longtemps pour augmenter les chances de guérison.» 


\section{- La vie sociale}

La vie sociale reflète la manière dont les gens explorent leur environnement et s'adaptent aux contraintes, climatique et géographique. Une adaptation tant architecturale qu'urbaine ou rurale. La vie sociale avec ses traditions et culture constitue un fondement d'originalité et de spécificité tant recherché par les touristes.

\section{- La vie urbaine}

Les ksour - "... Dans le sud le touriste visitera des ksour très intéressants. Ces villes présentent un enchevêtrement inextricable de ruelles étroites et tortueuses que bordent des maisons couvertes en terrasses. Certains quartiers sont réservés aux Juifs, d'autres aux nègres. »

La rue - "...Des fontaines publiques, souvent décorées de mosaïques, sont l'œuvre pie d'un bienfaiteur de la ville ou d'un riche commerçant... d'autres cafés maures qui représentent le seul lieu de "sortie » de la population masculine.... De part et d'autre de la rue, des boutiques se succèdent, simples échoppes sans vitrines, exigües et encombrées de marchandises : piles de soieries, de tapis, cuivres, maroquinerie...»

Les Souk hebdomadaires - " ...tenus en pleine campagne rappellent en plus pittoresque les foires de la métropole. Le touriste ne manquera pas de s'y mêler à l'occasion. Les noms de Souk-El-Had, Souk-El-Arba, traduisent dans la toponymie le rôle de marché hebdomadaire de ces lieux-dits. »

\section{- La vie rurale}

«La plus grande partie de la population...vit dans les campagnes. Les berbères ruraux sont en majorité sédentaires. Ils cultivent leurs jardins, labourent leurs champs et récoltent leurs fruits. Les arabes,...souvent nomades, ne possèdent généralement pas d'habitation fixe. Leurs troupeaux, groupés pour la garde, ne sont que très rarement propriété collective»

La vie rurale reposait sur la particularité de la vie sédentaire, nomade ainsi que sur le cultivateur et ses méthodes. Elle dépendait aussi des éleveurs et des contraintes climatiques et géographique aux quelles ils faisaient face.

Des activités touristiques avec respect des données climatiques, de la vie rurale et de la qualité paysagère :

- Des retombées économiques, par une exploitation du paysage naturel.

- Echange de services écologiques : touriste/région.

- Distribution dans le temps et dans l'espace des activités touristiques, la chasse, les courses, les cures, les campements, les balades, les promenades dans les oasis. 
- La promotion de Biskra comme station curative, par sa chaleur douce et ses eaux bienfaisantes.

- Finalement la promotion de Biskra comme la plus grande oasis. Dans le tableau (4) nous avons synthétisé les relations tissées entre le paysage et le tourisme comme la première entreprise qui l'a utilisé.

\begin{tabular}{|c|l|l|l|l|l|l|l|}
\hline Le paysage & \multicolumn{2}{|c|}{ géographique } & \multicolumn{2}{c|}{ climatologique } & \multicolumn{2}{c|}{ Végétal } \\
\hline Physique & montagne & oueds & soleil & pluie & vents & palmier & $\begin{array}{l}\text { Agrosystème } \\
\text { oasien }\end{array}$ \\
\hline \multicolumn{7}{|c|}{ Temps/ usages } \\
\hline Sensoriel & Les campements/ & $\begin{array}{l}\text { La } \\
\text { chasse }\end{array}$ & les cures & Les soins & La vie rurale & $\begin{array}{l}\text { La vie urbaine } \\
\text { Les souks }\end{array}$ \\
\hline Symbolique & $\begin{array}{l}\text { Ecoles } \\
\text { coranique }\end{array}$ & Mosquées & Les vestiges & $\begin{array}{l}\text { Qualité } \\
\text { des dattes }\end{array}$ & Le commerce \\
\hline
\end{tabular}

Tab.4 - Synthèse de la relation paysage usage

Sur ce tableau et d'après les récits analysés, nous remarquons que le paysage est classé en trois types géographique, climatologique et végétal, les usagés ont valorisé ces paysages et ils ont intégré des valeurs sensorielles et symboliques.

\section{Conclusion}

L'objectif de cet apport est de relire les récits de voyage sur le paysage oasien dans le but de savoir quels sont les éléments visuels et sensoriels qui définissent sa representation mentale. Certe, le paysage oasien ne se réduit pas aux données visuelles dans les récits de voyage étudiés. Il est toujours spécifié de quelque manière que ce soit par la subjectivité des auteurs. «L'étude paysagère est donc autre chose qu'une morphologie également de l'environnement. Inversement, le pays n'est pas uniquement « le miroir de l'âme ». Il se rapporte à des objets concrets. L'étude paysagère est autre chose qu'une psychologie du regard ...» (Augustin B., 1994)

- Un paysage hors échelle des usagés

Cependant, nous constatons que dans les périodes d'avant le colonialisme, les interventions humaines sur le paysage se faisaient de manière spontanée. 
Ces interventions ne façonnaient pas le paysage, elles n'ont le souci ni de le détruire (intentionnellement) ni de le bâtir ou de le préserver. Toutes les interventions s'insèrent dans le paysage comme une entité.

De ce fait les paysages n'étaient pas modifiés mais ponctués par des éléments visuels et sensoriels. Les éléments visuels physiques sont ou linéaires, comme le limes, les routes, les oueds, les seguias ou ponctuels comme les bâtissent ou les établissements humains. Quand aux éléments sensoriels, ils reflètent tout usage, activité et fonction, comme ils reflètent tout attachement, religieux, culturel ou social.

Un équilibre écologique défini la relation entre population, terre agraire, palmeraie et bâti. Ce qui produit une sorte de charte écologique entre population et environnement. A ce niveau la nous constatons que l'échelle du paysage est très importante par rapport à l'homme et son environnement construit ou vécu.

- Un usage paysager basé sur l'échange de services

Durant la période coloniale, une nouvelle fonction plus ou moins étrangère au contexte naturel et social s'insert. Il s'agit du " tourisme ". Que le paysage représente la nature, la vie sociale, la culture agricole ou le paturage, le tourisme pendant la période coloniale apparaissait à certain degré, amical avec le paysage oasien.

La chasse se faisait de manière à préserver les relations écologiques. Telle la relation chasseurs fellahs contre les oiseaux qui gênent. Les fellahs ouvraient grand les portes de leurs jardins aux chasseurs, des sangliers, des loups et des renards qui affectent les terres et les produits agraires et même les animaux d'élevages : moutons, chèvres.

Les animaux chassés mangeables se vendaient aux hôtels qui les utilisaient dans la restauration.

Dans un autre récit de voyage la chasse de gazelle se ferme pendant 3 ans pour laisser le temps aux gazelles de se procréer.

\section{- Temps /espaces /activités}

Un principe de la répartition des activités touristiques a surgi en tenant compte du paysage existant et de la capacité spatiale. Durant cette période il n'y a pas eu d'intervention sur le paysage et ses caractéristiques oasiennes; même les contraintes du site, tel les vents, la température étaient considérés comme atout. Il est bien évident que tout a été laissé à l'état sauvage.

Le flux des touristes était limité dans une période bien définie les 6 à 7 mois de l'année. La gamme des touristes était sélectionnée selon les caractéristiques climatiques et les ambiances thermiques. Il y avait : 
- De simples touristes,

- Des artistes, écrivains, journalistes, surtout peintres,

- Des retraités et des personnes fuyants le climat froid et humide de leur pays et à la recherche d'un climat plus favorable à leur santé. L'exploitation touristique du paysage, a proposé des parcours au cœur des sites historiques et anciens pour remonter dans le passé et des itinéraires de découvertes du patrimoine architectural et des monuments mis en scène, à travers des prospectus d'agences de voyage qui éveillent et justifient le désir d'évasion, ou suggèrent différents types de dépaysement, ou carrément proposent des structures sécurisantes.

L'usage des paysages dans l'aménagement a de multiples impacts: culturel, environnemental, social et économique. Leur connaissance approfondie nous offre des outils pour réaliser des projets de paysages sensibles, poétiques, harmonieux, au lieu de subir des aménagements et de découvrir tardivement le résultat d'un processus aléatoire susceptible de générer des images ébauchées et simplistes.

\section{Références bibliographiques}

ALKAMA Dj., (1999), « vers une nouvelle approche de la sauvegarde de l'équilibre urbain dans les oasis », in Seminaire Biskra. Pp 354-360.

Alkama Djamel, (2006) Pour Une Nouvelle Approche D'urbanisation Dans Les Zones Arides Cas Du Bas Sahara Les Ziban, le Souf et le Oued Righ . Thèse de doctorat soutenue à l'université Mohamed Kheider à Biskra.

Augustin Berque, Cinq propositions pour une théorie du paysage, 1994

Biskra guide vert, 1956

Bouzahar L. S. (mars, 2015), " Un aménagement durable par un projet écotouristique ; Cas des ksour de la micro région des Ziban. Le redressement d'un circuit écotouristique. » Thèse de doctorat soutenue à l'université Mohamed Kheider à Biskra.

Bouzahar L. S. (September, 2011), «The Ziban as Sustainable City in the Sahara» in proceedings CISBET Lausanne, Switzerland , pp.653-658.

Bouzahar L. S. (2009). In Les circuits touristiques, vecteur de préservation et d'intégration du patrimoine culturel. Le cas des Ksour et Dachra des Ziban In le séminaire international sur La Conservation Du Patrimoine: Didactiques Et Mise En Pratique. In October In Constantine, Algérie.

Bouzahar L. S. (2008) : Return to palm plantation of Ziban as Element of sustainable urban organization. In International Seminar On Sustainable Building Design “SBD08", Liverpool, U.K. July 
Carte Michelin $n^{\circ} 172$ - plis 17 et 18

Cardinal Lavigerie, 1956.

Colonel DELARTIGUE 1904,pp 5-9

Colonel Niox : pp 38-39, 1890,

Cote M., (1993) : L'Algérie ou l'espace retourné. Média-Plus, Algérie, Constantine, Algérie.

COURNOYER C., 2004, le paysage de l'oasis dans le sud du Maroc. Université de Montréal, Chaire UNESCO.

Direction des forêts, 2011

Direction du transport, 2011.

L'Abbe Jean Hurabielle, (1898) «Au pays du Bleu, BISKRA et les oasis environnantes » , Ed. Augustin challamel, Paris. Pp 215.

Gabriel Wackermann, (2005), Ville et environement. Ed. Ellipses pp.179-203.

Monographie de la wilaya de Biskra.

Rapport d'expertise de L'Agence National pour le Développement de l'Investissement, (2013) 